\title{
Constrained Multi-Objective Antenna Design Optimization using Surrogates
}

\author{
Prashant Singh*, Marco Rossi, Ivo Couckuyt, Dirk Deschrijver, Hendrik Rogier and \\ Tom Dhaene
}

Ghent University - iMinds, iGent Technologiepark-Zwijnaarde 15, 9052 Gent, Belgium.

\begin{abstract}
SUMMARY
A novel surrogate-based constrained multi-objective optimization algorithm for simulation-driven optimization is proposed. The evolutionary algorithms usually applied in antenna design optimization typically require a large number of objective function evaluations to converge. The Efficient Constrained Multi-objective Optimization (ECMO) algorithm described in this paper identifies Pareto-optimal solutions satisfying the required constraints using very few function evaluations. This leads to substantial savings in time and drastically reduces the time-to-market for expensive antenna design optimization problems. The efficiency of the approach is demonstrated on the design of an L1-band GPS antenna. The algorithm automatically optimizes the antenna geometry, parametrized by five design variables with performance constraints on three objectives. The results are compared with well-established multi-objective optimization evolutionary algorithms. Copyright (C) 2010 John Wiley \& Sons, Ltd.
\end{abstract}

Received ...

KEY WORDS: GPS antenna; Constrained Optimizaiton; Multiobjective Optimization; Surrogate Models

\section{INTRODUCTION}

Engineering optimization problems, such as the design of electronic filters and antennae, involve computationally expensive electromagnetic field simulations. Using Multi-Objective Evolutionary Algorithms (MOEAs) and genetic algorithms $[1,2,3,4,5,6]$ is not desirable, since they typically require a large number of objective function evaluations during the optimization process.

Surrogate-assisted algorithms have gained popularity in recent years for the problem of optimizing antennas. The approaches proposed in [7, 8, 9] use low-fidelity simulations to supplement (and minimize the number of) high fidelity simulations required during the optimization process. The approaches involve training a Kriging surrogate model by performing carefully chosen simulations according to a sampling algorithm. The Kriging model is then optimized using a MOEA. Since the Kriging model is substantially cheaper to evaluate than the computationally expensive objective function, the optimization process is expedited. The techniques of frequency scaling and additive response correction are applied in [10] to iteratively refine a surrogate model trained using coarse simulations. All the surrogate-assisted techniques described above involve training a surrogate model by carefully performing simulations at well-chosen points. The surrogate model is optimized in turn using an evolutionary algorithm. This scenario is termed as surrogate modeling.

Surrogate-Based Optimization (SBO) is distinct from surrogate modeling. It is a popular choice to expedite complex optimization problems $[11,12,13]$ involving expensive simulations. SBO involves generating surrogate models of the underlying system (e.g., a simulation model) on the fly.

*Correspondence to: prashant.singh@intec.ugent.be 
These models are trained by samples selected using a sampling algorithm. The sampling algorithm is designed with the objective of seeking the optima, or driving the search towards optimal regions in the design space. Statistical criteria such as probability of improvement (PoI), probability of feasibility (PoF) and expected improvement (EI) [14] utilize the mean and variance of prediction from the surrogate model, and are often used to solve single-objective optimization problems on a budget. Multi-objective formulations of PoI and EI [15] have been proposed, which can solve multiobjective optimization problems involving up-to 7 objectives. An advantage of SBO approaches is that they can economize on the number of objective function evaluations needed, as compared to surrogate modeling and evolutionary approaches. It is found that optimizing the objective functions directly requires less number of evaluations than would be needed to chart the entire optimization surface. This letter demonstrates how SBO can speed-up the overall design optimization of an antenna significantly and compares it with existing methods. The algorithm uses multiobjective formulations of the $\mathrm{PoI}$ and $\mathrm{PoF}$ criteria for expediting the constrained multi-objective design optimization. The details of the algorithm and its specific advantages in the efficient optimization of antennas are elucidated in Section 2. Section 3 then demonstrates the usefulness of the approach on a representative antenna design. Details about the applied algorithm are given in Section 5, whereas the outcome of the optimization process and pertinent conclusions are discussed in Sections 5 and 6 , respectively.

\section{EFFICIENT CONSTRAINED MULTI-OBJECTIVE OPTIMIZATION (ECMO)}

The EMO algorithm [15] very efficiently solves multi-objective optimization problems up to 7 objectives. The ECMO algorithm [16] extends the EMO algorithm with the ability to handle computationally expensive constrained multi-objective optimization problems. The sampling scheme in the original formulation of ECMO selects only one new sample per iteration. In this letter, the ECMO algorithm in [16] is extended such that multiple new candidate samples are selected per iteration, and an ensemble of heterogeneous surrogate models is utilized to aid the sample selection process.

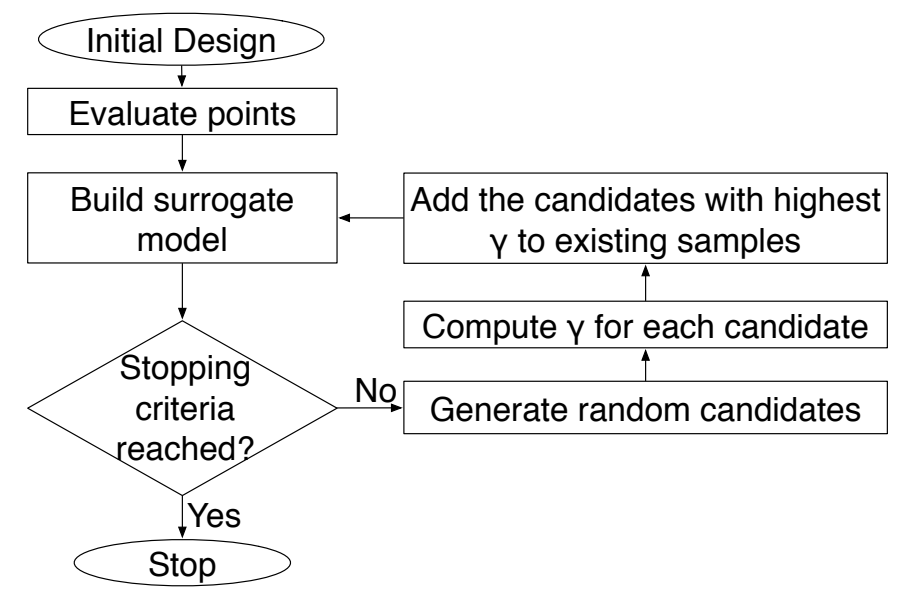

Figure 1. Flowchart of the ECMO algorithm.

The flowchart of the ECMO algorithm is shown in Fig. 1. The algorithm begins with a small set of samples known as the initial design, for which the objective functions are evaluated. The resulting training set is used to build a surrogate model. A cycle that selects new samples, evaluates them and subsequently re-trains the surrogate based on the updated training set continues until a specified stopping criterion (e.g., simulation budget, computation time, etc.) is met. The goal of sampling algorithms is to rapidly drive the search towards the optima. The ECMO algorithm employs two sampling criteria to quickly and efficiently identify a Pareto-optimal set of solutions that satisfy 
the specified constraints. The following subsections explain the hypervolume based Probability of Improvement and Probability of Feasibility used as sampling criteria in the ECMO algorithm.

Hypervolume-based Probability of Improvement (PoI) The expected improvement and probability of improvement are popular sampling criteria for single-objective optimization problems. For multi-objective optimization problems, the optimal solutions are represented by a Pareto set of trade-offs between the objectives. One way to measure improvement over an intermediate Pareto set is to use the hypervolume-based PoI, which has been shown to be fast and reliable [15]. The hypervolume-based PoI is defined as

$$
P_{h v}[I(\mathbf{x})]=\mathcal{H}_{\text {contr }}(\mathbf{x}) \times P[I(\mathbf{x})],
$$

where $\mathcal{H}_{\text {contr }}(\mathbf{x})$ is the contributing hypervolume measuring the improvement of a new sample $\mathbf{x}$ over the Pareto set and $P[I(\mathbf{x})]$ is the multi-objective PoI defined as

$$
P[I(\mathbf{x})]=\int_{\mathbf{y} \in A} \prod_{j=1}^{m} \phi_{j}\left(y_{j}\right) d y_{j},
$$

with $A$ the non-dominated region of the objective space and $m$ the number of objective functions. The function $\phi_{j}$ is the probability density function associated to the surrogate model (e.g., Kriging, Radial Basis Function (RBF), Support Vector Regression (SVR)) for the $j^{\text {th }}$ objective denoted as

$$
\phi_{j}\left(y_{j}\right) \triangleq \phi_{j}\left[y_{j} ; \hat{y}_{j}(\mathbf{x}), s_{j}^{2}(\mathbf{x})\right]
$$

Probability of Feasibility (PoF) The PoF criterion measures the probability of a sample satisfying the constraints. Assuming $k$ constraint functions, each modelled by a surrogate model, the probability of the prediction being greater than a specified constraint limit is computed in a manner similar to the probability of improvement. Let $\hat{g}^{i}(\mathbf{x})$ be the prediction and $s_{i}^{2}(\mathbf{x})$ the variance of the surrogate model for the $i^{\text {th }}$ constraint. The probability of feasibility can then be defined as

$$
P\left[F_{i}(\mathbf{x})>g_{\min }^{i}\right]=\frac{1}{s \sqrt{2 \pi}} \int_{0}^{\infty} e^{\frac{-\left(F_{i}(\mathbf{x})-\hat{g}^{i}(\mathbf{x})\right)^{2}}{2 s^{2}}} d G_{i}(\mathbf{x}),
$$

with $g_{\min }^{i}$ being the limiting constraint value, $F_{i}(\mathbf{x})=G_{i}(\mathbf{x})-g_{\min }^{i}$ the measure of feasibility and $G_{i}(\mathbf{x})$ a random variable for the $i^{t h}$ constraint. The combined probability of feasibility of satisfying $k$ constraints then becomes

$$
P_{c}(\mathbf{x})=\prod_{i=1}^{k} P\left[F_{i}(\mathbf{x})>g_{\text {min }}^{i}\right] .
$$

The final criterion $\gamma$ applied in this work is obtained by multiplying the hypervolume-based PoI with the PoF as

$$
\gamma(\mathbf{x})=P_{h v}[I(\mathbf{x})] \times P_{c}(\mathbf{x}) .
$$

Optimizing this criterion results in selecting points that improve the Pareto set satisfying all constraints, while also improving the accuracy of the surrogate models. $\gamma$ is optimized using a hybrid Monte-Carlo-based approach for experiments performed in this work.

Ensemble model construction and selection For many applications, the most appropriate surrogate model type is not known beforehand. As the evaluation of the objectives by electromagnetic field simulations is much more expensive than the computational cost of training models, an ensemble-based approach is needed to reduce the burden of evaluating the best model type using repeated runs. Therefore, an ensemble of multiple surrogates (e.g., Kriging, SVR, RBF) is trained in each iteration of the ECMO algorithm. A cross-validation step determines the most accurate surrogate, which is then used in conjunction with the sampling criteria. 


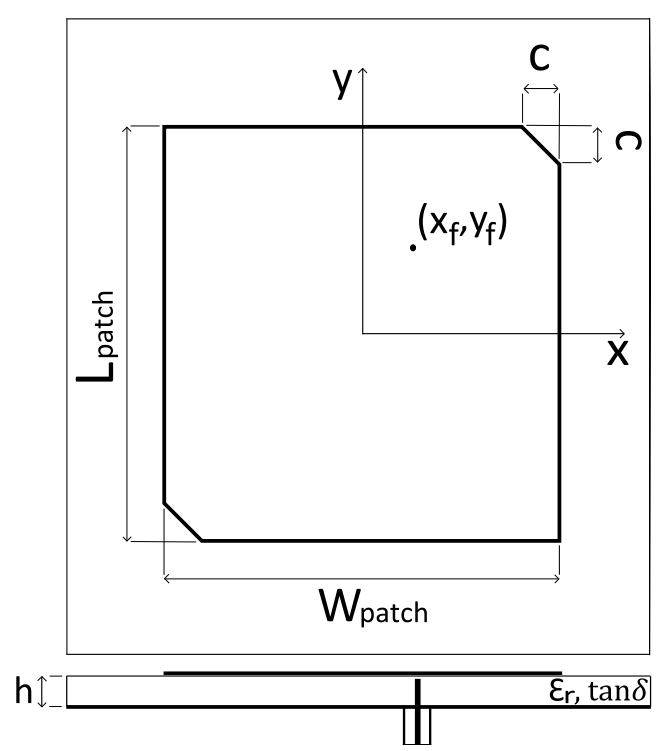

Figure 2. Representative textile microstrip probe-fed GPS patch antenna.

\section{OPTIMIZATION OF A GPS ANTENNA}

Consider a textile microstrip probe-fed compressible GPS patch antenna [17], as shown in Fig. 2. This antenna consists of a square patch with two truncated corners glued on a flexible closed-cell expanded rubber protective foam substrate. The patch is fed in the top right corner by a coaxial probe, exciting a right hand circular polarization. The nominal characteristics of the substrate are relative permittivity $\epsilon_{r}$ equal to 1.56 , loss tangent $\tan \delta$ equal to 0.012 and thickness $h$ equal to 3.94 $\mathrm{mm}$.

The optimization of the design of such a GPS antenna is a nontrivial task, as multiple constraints have to be satisfied. First, the antenna has to comply with the requirements of the GPS-L1 standard. Therefore, its return loss $\left|S_{11}\right|$ has to be lower than $-10 \mathrm{~dB}$ and its axial ratio AR (defined as the ratio between the amplitudes of the orthogonal components composing the circularly polarized field) has to be smaller than $3 \mathrm{~dB}$ in the $[1.56342,1.58742] \mathrm{GHz}$ frequency band. Second, the fulfilment of these criteria has to be achieved without sacrificing the directive gain of the antenna, which is of paramount importance for its correct operation. Moreover, since the antenna is simulated by means of the Keysight's ADS Momentum 2012-08 full-wave solver, the whole process is expected to be very time consuming. As a result, the optimization of the design of the antenna may largely benefit from the advocated algorithm.

Therefore, the ECMO algorithm is applied to optimize the considered design with respect to its $\left|S_{11}\right|$, boresight AR and boresight Gain in the GPS-L1 frequency band. More specifically, the objectives of the optimization are minimizing $\left|S_{11}\right|_{\max }$ and $\mathrm{AR}_{\max }$, and maximizing Gain. The constraints are:

$$
\begin{gathered}
\mathrm{AR}=\mathrm{AR}_{\text {max }}-\mathrm{AR}_{\text {lim }} \\
\left|S_{11}\right|=\left|S_{11}\right|_{\text {max }}-\left|S_{11}\right|_{\text {lim }}
\end{gathered}
$$

where the limits $\mathrm{AR}_{\text {lim }}$ and $\left|S_{11}\right|_{\text {lim }}$ are dictated by the GPS-L1 standard, being $3 \mathrm{~dB}$ and $-10 \mathrm{~dB}$, respectively. $\mathrm{AR}_{\max },\left|S_{11}\right|_{\max }$ and $\mathrm{Gain}_{\min }$ are the maximum and the minimum values, respectively, at operating frequencies $1.56342 \mathrm{GHz}, 1.57542 \mathrm{GHz}$ and $1.58742 \mathrm{GHz}$. All the geometric parameters of the antenna are considered in the optimization process, their variation ranges being: 


$$
\begin{aligned}
72.6 \mathrm{~mm} & <L_{\text {patch }}<75.2 \mathrm{~mm}, \\
69.2 \mathrm{~mm} & <W_{\text {patch }}<71.5 \mathrm{~mm}, \\
6.5 \mathrm{~mm} & <x_{f}<9.7 \mathrm{~mm}, \\
13.8 \mathrm{~mm} & <y_{f}<16.4 \mathrm{~mm}, \text { and } \\
3 \mathrm{~mm} & <c<6 \mathrm{~mm} .
\end{aligned}
$$

\section{NUMERICAL SETTINGS}

All experiments were performed using the SUMO Toolbox [11] for MATLAB $\AA$, which is freely available for non-commercial use. The initial design is a Latin Hypercube of 100 points, in addition to the 32 corner points. An ensemble of Kriging, RBF and SVR models is trained using the ECMO algorithm. The ECMO algorithm selects 5 new points in each iteration, until the simulation budget of 250 points is exhausted. Each simulation takes approximately one minute on an Intel Core i5 machine with 4 GB RAM.

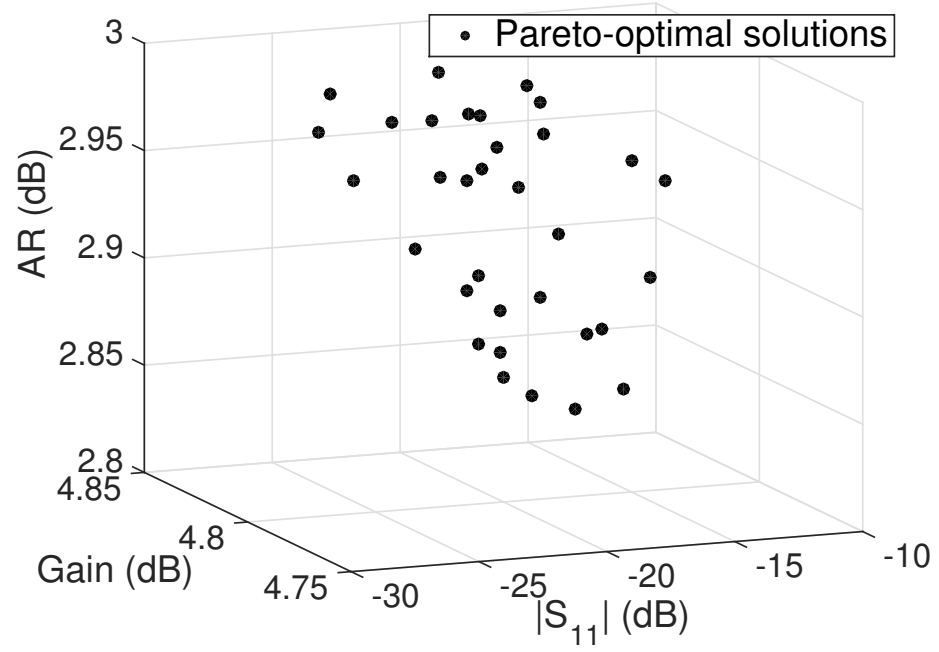

Figure 3. GPS Antenna: Pareto set of solutions satisfying constraints, obtained using the ECMO algorithm.

Each point is a 5-dimensional vector $\mathbf{x}=\left\{\mathbf{L}, \mathbf{W}, \mathbf{c}, \mathbf{x}_{\mathbf{f}}, \mathbf{y}_{\mathbf{f}}\right\}$ corresponding to a realization of the GPS antenna under study, which is then simulated in Keysight's ADS Momentum 2012-08 to evaluate the objectives and constraints (Eqs. 7, 8).

\section{RESULTS AND DISCUSSION}

The results of the surrogate-based optimization are plotted in Fig. 3. They are compared against the NSGA-II [18] and SMS-EMOA [19] multi-objective evolutionary algorithms (MOEAs) on the hypervolume metric (Fig. 4). Online convergence detection [20] was enabled for SMS-EMOA. Support for constraints was enabled for all algorithms. The hypervolume of the intermediate Pareto sets obtained using ECMO are consistently better than from NSGA-II and SMS-EMOA. This translates into solutions that are diverse and present a wider choice for the practitioner. A Pareto set of 33 solutions was obtained using the ECMO algorithm. All of them satisfy the constraints specified by the GPS-L1 standard. A possible way to choose between Pareto-optimal solutions is to consider the AR values, which is the most crucial parameter in the design of the GPS-L1 


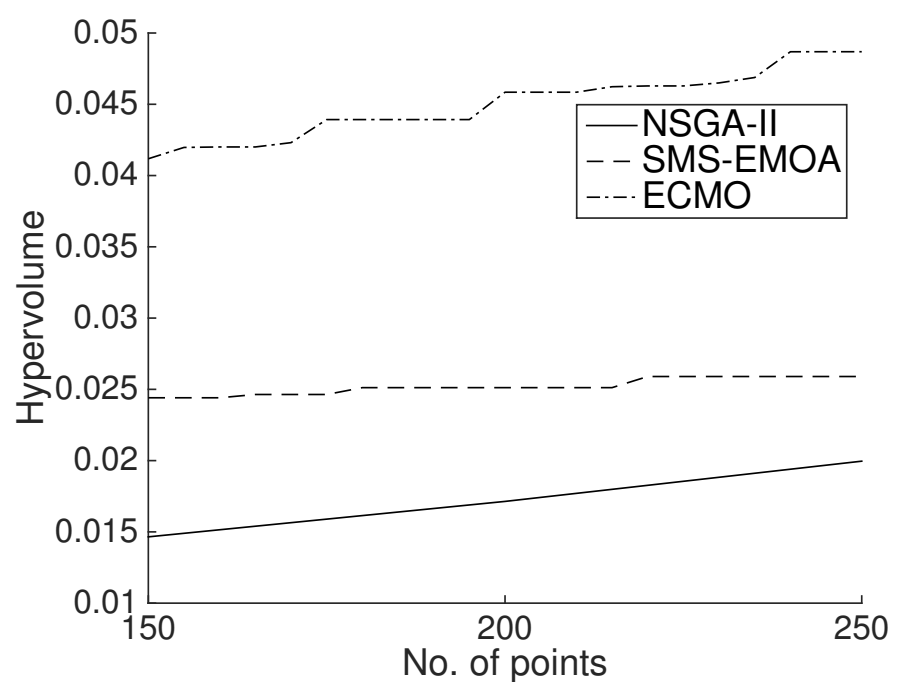

Figure 4. GPS Antenna: Evolution of the hypervolume metric for NSGA-II, SMS-EMOA and ECMO.

compatible antenna. The chosen solution, therefore, is $\{74.1250,69.7676,3.2995,7.9560,16.4306\}$ having $\left|S_{11}\right|$, Gain and AR values $\{-15.8593,-4.8162,2.8317\}$ respectively.

Although the ensemble-based model construction scheme adds some computational overhead, it is small compared to the overall cost of performing a simulation. The advantage of the algorithm is the ability to solve constrained multi-objective optimization problems using very few objective function evaluations. The total time taken by the ECMO algorithm for the optimization process was $\approx 5$ hours, as opposed to $\approx 30$ to 40 hours for the MOEAs.

\section{CONCLUSION}

The Efficient Constrained Multi-objective Optimization (ECMO) algorithm was proposed to solve constrained multi-objective antenna design optimization problems involving expensive electromagnetic field simulations. The algorithm combines surrogate models such as Kriging, Radial Basis Functions and Support Vector Regression along with hypervolume Probability of Improvement $(\mathrm{PoI})$ and Probability of Feasibility $(\mathrm{PoF})$ sampling criteria to efficiently drive the search towards optimal solutions. The algorithm is applied to optimize an L1-band GPS antenna. Results show that ECMO outperforms state-of-the-art Multi-Objective Evolutionary Algorithms (MOEAs) such as NSGA-II and SMS-EMOA, and offers substantial savings in time.

\section{ACKNOWLEDGEMENT}

This work was supported by the Interuniversity Attraction Poles Programme BESTCOM initiated by the Belgian Science Policy Office, and the Research Foundation Flanders (FWO- Vlaanderen). Ivo Couckuyt is a Postdoctoral Researcher of FWO-Vlaanderen.

\section{REFERENCES}

[1] Nguyen T, Morishita H, Koyanagi Y, Izui K and Nishiwaki S, A multi-level optimization method using PSO for the optimal design of an L-shaped folded monopole antenna array, IEEE Transactions on Antennas and Propagation, 2014, 62(1), pp. 206-215.

[2] Goudos S and Sahalos J, Pareto optimal microwave filter design using multiobjective differential evolution, IEEE Transactions on Antennas and Propagation, 2010, 58(1), pp. 132-144. 
[3] Bhattacharya S, Chattopadhyay S, Talukder S, Bag S, Mishra S and Gangopadhyaya M, Optimization of inset-fed microstrip patch antenna using genetic algorithm, International Conference and Workshop on Computing and Communication (IEMCON), 2015, pp. 1-4.

[4] Manh L, Grimaccia F, Mussetta M and Zich R, A Soft Computing Hybridization Technique for Antenna Optimization, International Journal on Communications Antenna and Propagation (IRECAP), 2015 5(1), pp. 16-20.

[5] Dierck A, Declercq F, Vervust T and Rogier H, Design of a circularly polarized Galileo E6-band textile antenna by dedicated multi-objective constrained Pareto-optimization, International Journal of Antennas and Propagation, 2015, Article ID 895963, doi:10.1155/2015/895963.

[6] Bianchi D, Genovesi S and Monorchio A, Constrained Pareto optimization of wide band and steerable concentric ring arrays, IEEE Transactions on Antennas and Propagation, 2012, 60(7), pp. 3195-3204.

[7] Koziel S and Ogurtsov S, Multi-objective design of antennas using variable-fidelity simulations and surrogate models, IEEE Transactions on Antennas and Propagation, 2013, 61(12), pp. 5931-5939.

[8] Koziel S, Bekasiewicz A and Zieniutycz W, Expedited EM-driven multiobjective antenna design in highly dimensional parameter spaces, IEEE Antennas and Wireless Propagation Letters, 2014, 13, pp. 631-634.

[9] Koziel S, Bekasiewicz A, Couckuyt I and Dhaene T, Efficient multi-objective simulation-driven antenna design using Co-Kriging, IEEE Transactions on Antennas and Propagation, 2014, 62(11), pp. 5900-5905.

[10] Koziel S, Bekasiewicz A and Zieniutycz W, Design of a planar UWB dipole antenna with an integrated balun using surrogate-based optimization, IEEE Antennas and Wireless Propagation Letters, 2014, 14, pp. 366-369.

[11] Gorissen D, Couckuyt I, Demeester P, Dhaene T and Crombecq K, A Surrogate Modeling and Adaptive Sampling Toolbox for Computer Based Design, The Journal of Machine Learning Research, 2010, 11, pp. 2051-2055.

[12] Wang G and Shan S, Review of metamodeling techniques in support of engineering design optimization, Journal of Mechanical design, 2007, 129(4), pp. 370-380.

[13] van der Herten J, Couckuyt I, Deschrijver D and Dhaene T, Adaptive classification under computational budget constraints using sequential data gathering, Advances in Engineering Software, 2016, 99, pp. 137-146.

[14] Forrester A and Keane A, Recent advances in surrogate-based optimization, Progress in Aerospace Sciences, 2009, 45(1), pp. 50-75.

[15] Couckuyt I, Deschrijver D and Dhaene T, Fast calculation of multiobjective probability of improvement and expected improvement criteria for Pareto optimization, Journal of Global Optimization, 2014, 60(3), pp. 575-594.

[16] Singh P, Couckuyt I, Ferranti F and Dhaene T, A constrained multi-objective surrogate-based optimization algorithm, IEEE Congress on Evolutionary Computation (CEC), 2014, 3, pp. 3080-3087.

[17] Vallozzi L, Vandendriessche W, Rogier H, Hertleer C and Scarpello, M, Design of a protective garment GPS antenna, Microwave and Optical Technology Letters, 2009, 51(6), 2009, pp. 1504-1508.

[18] Deb K, Pratap A, Agarwal S and Meyarivan T, A fast and elitist multiobjective genetic algorithm: NSGA-II, IEEE Transactions on Evolutionary Computation, 2002, 6(2), pp. 182-197.

[19] Beume N, Naujoks B and Emmerich M T M, SMS-EMOA: Multiobjective selection based on dominated hypervolume, European Journal of Operational Research, 2007, 181(3), pp. 1653-1669.

[20] Wagner T and Trautmann H, Online convergence detection for evolutionary multi-objective algorithms revisited, IEEE Congress on Evolutionary Computation (CEC), 2010, 3, pp. 1-8. 